\title{
Regionale Wirkungen von Maßnahmen zur Verbesserung des Ausbildungsangebotes
}

\section{Einleitung, Problemstellung}

Einer Verbesserung des Ausbildungsangebotes in ländlichen, wirtschaftlichen Problemgebieten liegen hauptsächlich zwei Zielsetzungen zugrunde: Angestrebt wird einerseits eine im Vergleich zu wirtschaftlich starken Regionen gleichwertige Versorgung mit Ausbildungseinrichtungen. Dieses Ziel orientiert sich am zentralen bildungspolitischen Postulat der Verringerung der Chancenungleichheit, das in der Forderung, Kinder sollten nicht aufgrund ihrer regionalen und sozialen Herkunft, sowie aufgrund ihres Geschlechts, ungleiche Bildungschancen haben, in knapper Form ausgedrückt werden kann. Andererseits bestimmt der Ausbildungssektor durch seinen Einfluß auf die Qualität des Arbeitskräftepotentials die wirtschaftlichen Entwicklungschancen einer Region entscheidend mit. Gut ausgebildete Arbeitskräfte der verschiedenen Qualifikationsstufen bilden eine wesentliche Voraussetzung, um die Attraktivität einer Region für expansionswillige Wirtschaftsunternehmen zu erhöhen und damit einen wirtschaftlichen Entwicklungsprozeß in die Wege zu leiten. Beide Zielsetzungen (Verbesserung der Versorgung mit Ausbildungseinrichtungen, Erhöhung des Angebotes an qualifizierten Arbeitskräften) lassen sich dem übergeordneten regionalpolitischen Hauptziel unterordnen, das eine Verringerung der interregionalen Einkommens- und Wohlfahrtsunterschiede anstrebt (FREY 1979, S. 33).

Die regionalen Wirkungen von Maßnahmen zur Verbesserung des Ausbildungsangebotes wurden bisher jedoch nur in wenigen Arbeiten untersucht, so daß bis heute zu wenig gesicherte Ergebnisse über die Zieleffizienz von Bildungsinvestitionen vorliegen. Dieser Artikel will einen Beitrag zu einer modellmäßigen Klärung der regionalen Auswirkungen von Bildungsinvestitionen leisten. ${ }^{1)}$ Die folgenden Ausführungen orientieren sich an den Verhältnissen in peripheren, wirtschaftlich benachteiligten Regionen innerhalb der westlichen Industrieländer (z. B. große Teile des Alpenraumes) und beziehen sich hauptsächlich auf Investitionen zur Verbesserung des Ausbildungsangebotes im nachobligatorischen Ausbildungsbereich der verschiedenen Stufen und Typen. ${ }^{2)}$

Abbildung 1 stellt eine schematische Übersicht über die wichtigsten, von Maßnahmen zur Verbesserung des Ausbildungsangebotes beeinflußten Variablen, dar. Diese werden in den folgenden Kapiteln näher erläutert.

\section{Beeinflussung des Bildungsverhaltens: Zunahme der Bildungsbeteiligung}

Periphere Regionen sind im allgemeinen durch das Fehlen gewisser weiterführender Schultypen (Indikator: Ausbildungsplätze/Kopf) sowie durch die schlechtere Erreichbarkeit des vorhandenen Ausbildungsangebotes für gewisse Teile der Bevölkerung (Indikator: Länge des Schulweges) gekennzeichnet (GEISSLER 1968, S. 244 ff., OECD 1979b, S. 155). Die schlechtere Erreichbarkeit der Ausbildungseinrichtungen drückt sich unter anderem in einer finanziellen Mehrbelastung der Familien aus, indem weite Schulwege entsprechend höhere Kosten für Anreise und auswärtige Unterkunft verursachen. Sie ist ein wesentlicher Bestimmungsfaktor für die niedrigere Bildungsbeteiligung (Anteil einer Altersgruppe, die eine Ausbildung absolviert) in ländlichen Regionen (MEUSBURGER 1976, S. 26).

Ein weiterer Bestimmungsfaktor der regionalen Bildungsbeteiligung ist die sozioökonomische Struktur der regionalen Bevölkerung, zu deren Kennzeichnung sich Merkmale wie Beruf, berufliche Stellung und Ausbildungsniveau eignen. Die enge Korrelation zwischen Bildungsbeteiligung der Kinder und der beruflichen Tätigkeit bzw. dem Ausbildungsniveau der Eltern ist in verschiedenen Untersuchungen festgehalten worden (z. B. GEIPEL 1969, S. 17, MEUSBURGER 1974, S. 17 ff.). Je niedriger das Ausbildungsniveau der Eltern bzw. deren berufliche Stellung, desto geringer ist die Bildungsbeteiligung der Kinder an weiterführenden Schulen. Furcht vor Schulversagen, Vorurteile gegenüber Wert und Nutzen weiterführender Bildungsgänge, Angst vor Entfremdung der Kinder sowie finanzielle Hindernisse sind dabei als wichtigste Hemmfaktoren zu betrachten (vgl. z. B. BAUER 1970, S. 11). Die geringere Bil-

Elisabeth Bühler-Conrad, Geographisches Institut Universität Zürich, 8033 Zürich. 


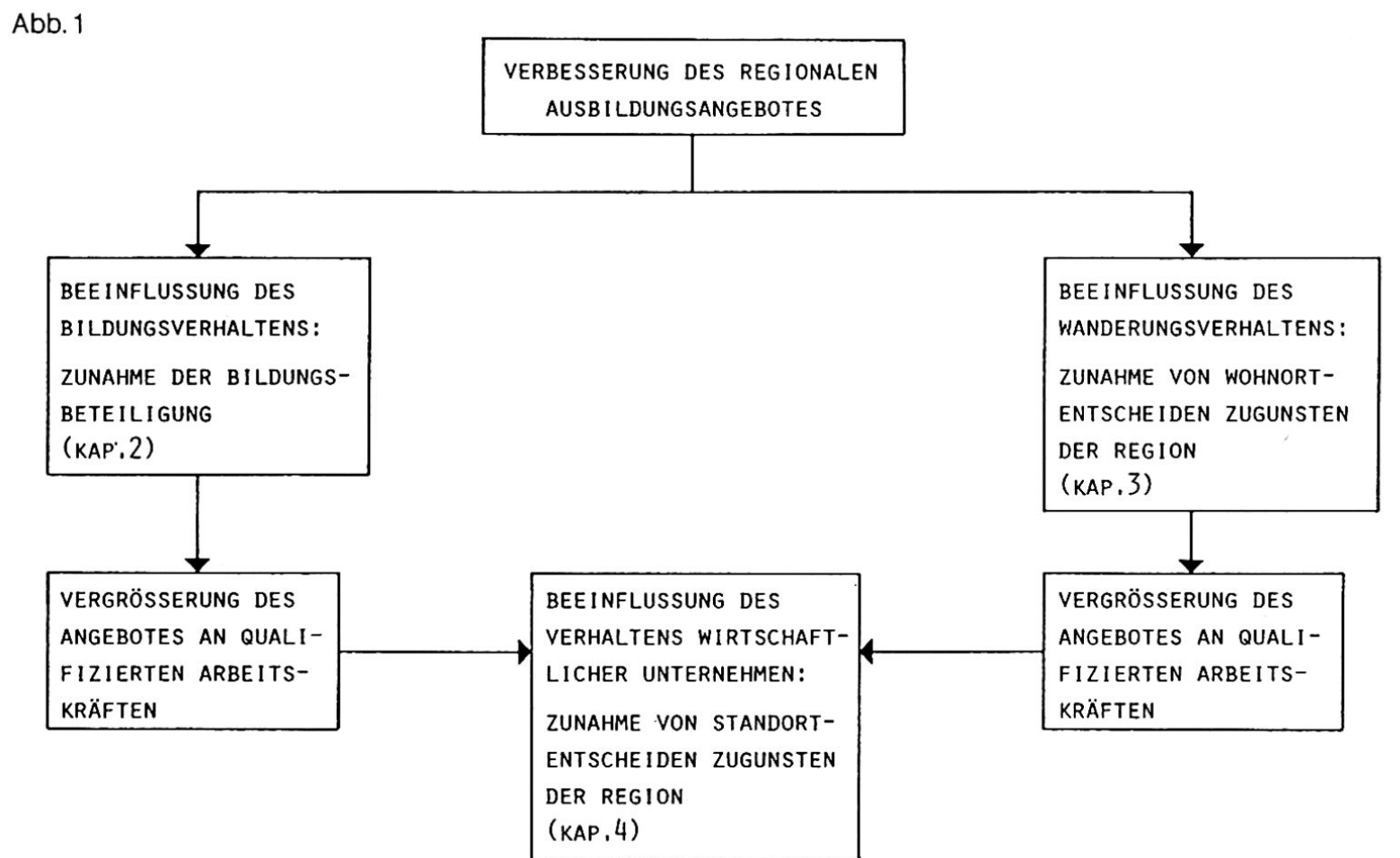

dungsmotivation sozial tieferer Schichten drückt sich auch in einer größeren Distanzempfindlichkeit der Bildungsbeteiligung aus: Jugendliche aus tieferen Bildungsschichten lassen sich vom Angebot an gut erreichbaren Ausbildungseinrichtungen stärker in ihrer Ausbildungsentscheidung beeinflussen als Jugendliche aus höheren Bildungsschichten (MEUSBURGER 1976, S. 32).

Eine Verbesserung des regionalen Ausbildungsangebotes stellt somit einen wichtigen Teilschritt in der Verwirklichung des Postulates gleicher Bildungschancen dar und hat eine Zunahme der Bildungsbeteiligung, insbesondere von Jugendlichen aus sozial tieferen Schichten, zur Folge. Die sozialen und geschlechtsspezifischen Ursachen der unterdurchschnittlichen Bildungsbeteiligung gewisser Bevölkerungsgruppen sind jedoch nur durch zusätzliche Maßnahmen der Bildungswerbung (Information, Beratung, finanzielle Unterstützung) zu beeinflussen (BAUER 1970, S. 10).

\section{Beeinflussung des Wanderungsverhaltens: Zunahme von Wohnortentscheiden zugunsten der Region}

Nach GATZWEILER (1975, S. 78) bilden die Ausbildungsmöglichkeiten den dominanten Bestimmungsgrund für die Abwanderung jugendlicher Personen aus peripheren Regionen («Bildungswanderer»). Diese Aussage wird auch durch die Resultate einer Wanderungsmotivbefragung bei weiblichen Personen aus dem schweizerischen Berggebiet von BUFF/ WALDER et al. (1978, S. 110 ff.) bestätigt. Mit einer Verbesserung des Ausbildungsangebotes kann deshalb die Abwanderung dieser Altersgruppe aus den peripheren Regionen entscheidend verringert werden.

Wie Ergebnisse empirischer Untersuchungen in der Bundesrepublik Deutschland zeigen, sind dagegen die Zuzüge in ländliche Gebiete, selbst aufgrund einer neuerrichteten Hochschule, vernachlässigbar gering (ENGELBRECH/KUEPPERS/SONNTAG 1977, S. 154).

Änderungen des Wanderungsverhaltens infolge eines verbesserten Ausbildungsangebotes sind jedoch nicht nur bei bildungswilligen Jugendlichen, sondern auch bei Haushalten mit Kindern zu erwarten. So schreibt beispielsweise STORBECK (1968, S.64), daß die Anwerbung auswärtiger Fach- und Führungskräfte und die Bemühungen, diese qualifizierten Mitarbeiter im Betrieb zu halten, durch ein gutes Bildungsangebot erheblich erleichtert werden, "weil diese Mitarbeiter neben anderen Bedingungen ein ausgebautes Bildungsangebot am Standort für ihre Kinder wünschen».

Aufgrund der bisherigen Ergebnisse der Wanderungsmotivforschung dürfen diese Effekte von 
neuen Schulen allerdings nicht überbewertet werden (vgl. z. B. HADERMANN/KAEPPELI/KOLLER 1975 und H.ZIMMERMANN 1975). Neben den beruflichen, wohnungsorientierten und persönlichen (z. B. Heirat) Bestimmungsgründen von Wanderungen kommt den Ausbildungsmöglichkeiten für die Kinder im Durchschnitt eine stark untergeordnete Bedeutung zu. Den größten Wert auf ein gutes Angebot an weiterführenden Schulen legten die durch das $\mathrm{Al}$ ter ihrer Kinder unmittelbar betroffenen 40-45jährigen befragten Personen (BULWIEN 1973, S. 136). Diese Altersgruppe weist jedoch andererseits eine mit Abstand geringere räumliche Mobilität auf als z. B. diejenige unter 35 Jahren (GATZWEILER 1975, S. 78), weshalb auch von ihr keine bedeutenden Wanderungsgewinne zu erwarten sind.

Eine Zusammenfassung der bisherigen Ausführungen zeigt, daß die Bereitstellung von besseren Ausbildungsmöglichkeiten in peripheren Regionen hauptsächlich das Verhalten der jugendlichen Bevölkerungsgruppen beeinflußt, indem sie die Bildungsbeteiligung erhöht und die Abwanderung zu Ausbildungszwecken reduziert. Als Folge dieser Effekte vergrößert sich das regionale Angebot an jungen qualifizierten Arbeitskräften. Eine signifikante Beeinflussung des Verhaltens anderer Bevölkerungsgruppen ist aufgrund der bestehenden Untersuchungen als wesentlich unwahrscheinlicher einzuschätzen.

\section{Beeinflussung des Verhaltens wirtschaftli- cher Unternehmen: Zunahme von Standort- entscheiden zugunsten der Region}

Nach ENGELBRECH/KUEPPERS/SONNTAG (1978, S. 14) wird die Relevanz von Bildungsinvestitionen für den wirtschaftlichen Entwicklungsprozeß erst sichtbar, wenn man den Einfluß von Größen wie «Bildungsbeteiligung», «regionales Arbeitskräfteangebot» oder "Wohnortgunst» usw. untersucht, den diese Größen auf Standortwahl und Entwicklungsmöglichkeiten der Betriebe bzw. auf Volumen und Struktur des regionalen Arbeitsplatzangebotes ausüben. Diese Relevanz von Ausbildungseinrichtungen für Betriebe hängt mit dem Bedarf an qualifizierten Arbeitskräften zusammen: einerseits und primär, weil gute Schulen auch gewährleisten, daß die Qualifikation des örtlichen Arbeitskräftepotentials gut ist und andererseits, weil ein gutes schulisches Angebot den Wohnortwünschen der qualifizierten Arbeitskräfte entgegenkommt (FUERST/ZIMMERMANN/HANSMEYER 1973, S. 109).

Aus empirischen Untersuchungen zum Standortwahlverhalten von Betrieben geht jedoch hervor, daß sowohl weiterführende allgemeinbildende als auch berufsbildende Schulen inkl. Hochschulen aus der Sicht des Unternehmers als praktisch bedeu- tungslos für den Standortentscheid in peripheren Regionen beurteilt werden (u.a. BULWIEN 1973, FREUND/ZABEL 1978, FUERST/ZIMMERMANN/HANSMEYER 1973). Diese Ergebnisse legen den Schluß nahe, daß Betriebe, für die das Angebot an höherqualifizierten Arbeitskräften ein wichtiger Standortfaktor ist, sich im allgemeinen nicht in wirtschaftsschwachen Regionen niederlassen. ENGELBRECH/ KUEPPERS/SONNTAG (1978, S. 151) sehen eine Ursache dieses Verhaltens im Fehlen gewisser hochwertiger, von diesen Betrieben stark nachgefragten Infrastrukturleistungen (zentrale Verwaltungsstellen, Bibliotheken, Flughäfen usw.) in peripheren Regionen. Daneben ist zu berücksichtigen, daß das Fehlen eines ausreichenden und differenzierten Angebotes an qualifizierten Arbeitskräften, ein typisches Merkmal wirtschaftsschwacher, dünn besiedelter Gebiete, zumindest kurzfristig durch den Ausbau des Ausbildungsangebotes nicht entscheidend verbessert werden kann.

Wie RAUM (1972, zitiert nach BUTTLER/GERLACH/ LIEPMANN 1975, S. 86) in einer empirischen Untersuchung feststellte, weisen Betriebe, die sich bevorzugt in peripheren Regionen niederlassen, eine Qualifikationsstruktur von Arbeitsplätzen auf, die durch einen hohen Bedarf an ungelernten Arbeitskräften, einen geringen Bedarf an Angestelltenberufen sowie einen nahezu unbedeutenden Bedarf an Arbeitskräften mit über Volksschulniveau liegender schulischer Qualifikation charakterisiert werden kann. Aufgrund der vorgestellten Untersuchungen muß deshalb die These zurückgewiesen werden, daß mit einer Verbesserung des Ausbildungsangebotes in peripheren Regionen deren Standortgunst signifikant erhöht wird bzw. die Zahl der attraktiven Arbeitsplätze bedeutend zunimmt. Eine Ausnahme stellen die durch zusätzliche Ausbildungseinrichtungen direkt geschaffenen Arbeitsplätze dar, da das Bildungswesen ein besonders beschäftigungsintensiver Infrastrukturbereich ist, dessen Arbeitsplätze zudem überdurchschnittlich krisensicher und qualitativ hochstehend sind. Nach GANSER (1978, S. 248) sind diese direkten Beschäftigungseffekte in wirtschaftlich benachteiligten Regionen auch quantitativ durchaus bedeutsam.

\section{Schlußfolgerungen}

Aus den bisherigen Ausführungen muß geschlossen werden, daß in peripheren Regionen als Folge der besseren Versorgung im Ausbildungsbereich bzw. der erhöhten Bildungsbeteiligung nicht alle Ausgebildeten in der Region adäquat beschäftigt werden können. Dieses Ungleichgewicht am regionalen Arbeitsmarkt (Überangebot an qualifizierten Arbeitskräften) kann durch berufliche Mobilität (Berufswechsel), räumliche Mobilität (Pendeln, Wegzug) 
oder durch eine Erhöhung der Arbeitslosenquote abgebaut werden (vgl. OECD 1979a, S. 33). Ergebnisse empirischer Untersuchungen zeigen, daß mit zunehmender Qualifikation die räumliche Mobilität steigt und die Bereitschaft zu einem Berufswechsel sinkt (vgl. z. B. HOFBAUER/NAGEL 1975, S. 259). Höherqualifizierte stellen im allgemeinen höhere Ansprüche an den Arbeitsplatz und sind deshalb eher bereit, aus beruflichen Gründen den Wohnort zu wechseln (HADERMANN/KOLLER/KAEPPELI 1975, S. 381). Zudem steigt mit zunehmendem Ausbildungsstand nicht nur das Anspruchsniveau an den Arbeitsplatz, sondern auch an die Versorgung mit privaten und öffentlichen Dienstleistungen (kulturelles Angebot, Einkaufsmöglichkeiten usw.), die in peripheren Regionen nur zum Teil gewährleistet ist (HADERMANN/KAEPPELI/KOLLER 1975, S. 562). Eine Abwanderung aus diesen Regionen wird dann erfolgen, wenn es nicht möglich sein wird, die größeren Arbeitsplatz- und Versorgungszentren durch tägliches Pendeln zu erreichen.

Bildungsinvestitionen bergen somit die Gefahr einer Verstärkung der Abwanderung aus wirtschaftsschwachen ländlichen Gebieten in sich. Solche Entleerungseffekte treten auf, weil das Arbeitsplatzangebot für höherqualifizierte Arbeitskräfte in den peripheren Regionen ungenügend ist und/oder weil mit einem höheren Ausbildungsstand ein höheres Anspruchsniveau an das öffentliche und private Dienstleistungsangebot einhergeht. Aus diesem Grund ist der Abbau der Abwanderung von Jugendlichen (vgl. Kap. 3) regionalpolitisch betrachtet nur als Teilerfolg zu werten (GANSER 1978, S. 245). Eine Verbesserung des Ausbildungsangebotes kann dazu beitragen, daß die peripheren Gebiete ständig einen Teil ihrer jungen, besser ausgebildeten Bevölkerung verlieren. Dieser Effekt ist als eine Verminderung der Entwicklungschancen einer Region zu beurteilen, da gerade diese Bevölkerungsgruppe ein tragendes wirtschaftliches und soziales Element im regionalen Entwicklungsprozeß darstellt. Unter dem Schlagwort "brain drain» ist die Migration qualifizierter Erwerbspersonen von wirtschaftlich schwachen in wirtschaftlich prosperierende Länder bekanntgeworden. Wie gezeigt werden konnte, ist dieses Phänomen jedoch auch innerhalb eines Landes zu beobachten.

Alternative regionalpolitische Strategien, welche dieser Tatsache Rechnung tragen, sind jedoch nicht in einem Verzicht auf den Ausbau des Bildungsangebotes in peripheren Regionen zu sehen. Einerseits weil ein solcher strategischer Ansatz mit dem wichtigen Ziel der Verwirklichung von mehr Chancengleichheit in Konflikt geraten würde und andererseits, weil damit übersehen würde, daß gut ausgebildete Arbeitskräfte nach wie vor eine wesentliche Voraussetzung für jede regionalwirtschaftliche Entwicklung darstellen. Vielmehr wären parallel und koordiniert mit der Verbesserung des Ausbildungsangebotes durch andere Maßnahmen der Wirtschaftsförderung in peripheren Regionen zusätzliche, attraktive Arbeitsplätze zu schaffen.

\section{Anmerkungen}

1) Diese Ausführungen stellen eine Zusammenfassung der theoretisch-methodischen Arbeit dar, die vorgängig zur empirischen Untersuchung im Rahmen eines Forschungsprojektes zum Thema "Auswirkungen von Infrastrukturinvestitionen», Teilbereich Bildung, geleistet wurde. Ausführlichere Ergebnisse sind enthalten in: HANSER CH./ BUEHLER-CONRAD E./KELLER TH.: Methodische Ansätze zur Wirkungskontrolle der Infrastrukturpolitik, Arbeitsbericht Nr. 11 des Nationalen Forschungsprogrammes «Regionalprobleme», Bern Mai 1980.

2) Das bedeutet allerdings nicht, daß sich nicht auch auf der Volksschulstufe in peripheren Regionen bedeutsame raumordnungspolitische Probleme stellen. Beispielhaft sei hier nur die Diskussion um die entwicklungspolitischen Vor- und Nachteile einer Zentralisierung der Schulstandorte in Gebieten mit geringer Bevölkerungsdichte und/ oder einem Bevölkerungsrückgang erwähnt (vgl. z. B. FROEHLICH 1977, MEUSBURGER 1976, UEHLINGER 1975).

\section{Literaturverzeichnis}

BAUER, HARTMUT: Räumliche Bestimmungsgründe der Situation im Bildungswesen als Grundlage regionaler Bildungsplanung, Mitteilungen aus dem Institut für Raumordnung, Heft 80, Bonn 1973.

BUFF, E. und WALDER, B. et al.: Migration der Frau aus Berggebieten. Geographisches und Soziologisches Institut der Universität Zürich, Zürich 1978.

BULWIEN, HARTMUT: Der Einfluß von Bildungseinrichtungen auf die unternehmerische Standortwahl, Geographische Diplomarbeit an der Technischen Universität München, München 1973.

BUTTLER, F., GERLACH, K. und LIEPMANN, P.: Funktionsfähige regionale Arbeitsmärkte als Bestandteil ausgeglichener Funktionsräume, in: Akademie für Raumforschung und Landesplanung, Forschungsund Sitzungsberichte, Band 94, Hannover 1975.

ENGELBRECH, G., KUEPPERS, G. und SONNTAG, J.: Regionale Wirkungen von Hochschulen, Bonn 1978.

FREUND, U. und ZABEL, G.: Regionale Wirkungen der Wirtschaftsförderung, Bonn 1978.

FREY, RENÉ L.: Die Infrastruktur als Mittel der Regionalpolitik, Bern 1979. 
FROEHLICH, HEIDI: Die schulische Versorgung in der Region Mittelbünden, Geographische Diplomarbeit, Geographisches Institut der Universität Zürich, Zürich 1977.

FUERST, D., ZIMMERMANN, K. und HANSMEYER, K.-H.: Standortwahl industrieller Unternehmer, Ergebnisse einer Unternehmerbefragung, Bonn 1973.

GANSER, KARL: Zur Raumbedeutsamkeit von Maßnahmen im Bildungsbereich, in: Akademie für Raumforschung und Landesplanung, Forschungsund Sitzungsberichte, Band 127, Hannover 1978.

GATZWEILER, HANS-PETER: Zur Selektivität interregionaler Wanderungen, Forschungen zur Raumentwicklung, Band 1, Bonn 1975.

GEIPEL, ROBERT: Bildungsplanung und Raumordnung als Aufgaben moderner Geographie, in: Geographische Rundschau 21, 1969.

GEISSLER, CLEMENS: Das Schulangebot aus regionaler Sicht, in: Bauwelt 1/2, 59. Jg., 1968.

HADERMANN, J., KAEPPELI, J. und KOLLER, P.: Räumliche Mobilität, Theoretische Grundlagen und empirische Untersuchung in der Planungsregion St. Gallen, St. Gallen 1975.

HOFBAUER, H. und NAGEL, E.: Regionale Mobilität bei männlichen Erwerbspersonen in der Bundesre- publik Deutschland, in: Mitteilungen aus der Arbeitsmarkt- und Berufsforschung, 6. Jg., Heft 3, Stuttgart 1973.

MEUSBURGER, PETER: Landes-Schulentwicklungsplan von Vorarlberg, Wien 1974.

MEUSBURGER, PETER: Entwicklung, Stellung und Aufgaben einer Geographie des Bildungswesens eine Zwischenbilanz, in: Mitteilungen der Österreichischen Geographischen Gesellschaft, Band 118, I, 1976.

OECD: L'enseignement et le développement régional, Rapport général, Volume I, Paris 1979a.

OECD: L'enseignement et le développement régional, Rapports techniques, Volume II, Paris 1979 b.

STORBECK, DIETRICH: Bocholt als regionales Bildungszentrum, Bedeutung und Wirkung einer höheren Wirtschaftsfachschule in der Stadt Bocholt, Materialien zur Raumplanung, 4, Münster 1968.

UEHLINGER, HEINER: Räumliche Aspekte der Schulplanung in ländlichen Siedlungsgebieten, Bern 1975. ZIMMERMANN, HORST: Räumliche Präferenzen: Wohnortwahl und Mobilitätsbereitschaft der Arbeitnehmer als Determinanten der Regionalpolitik, Bonn 1973.

\section{Literaturbesprechnung}

GEOGR. GESELLSCHAFT VON BERN (Hrsg.): Beiträge zun Klima des Kantons Bern. Bd 52/1975-76, 151 S., sep. 6 Karten und 2 Beilagen. Bern 1978, Fr. 30.--

Vorwiegend den Bedürfnissen der Raumplanung folgend, wurde im Jahre 1970 das Geographische Institut der Universität Bern (Prof. Dr.B. Messer1i) mit der Durchführung einer systematischen Klimaforschung als Grundlage für die Kantonsund Regionalplanung beauftragt. Nun wurden für 4 bis 5 Jahre drei jahresperiodische Naturerscheinungen erfasst und für den Kanton Bern kartographisch bearbeitet: phänologische Ereignisse als Merkmale der 3 Jahreszeiten Frühling (Apfelblüte), Hochsommer (Weizenernte) und Herbst (Blattverfärbung Buche), Bearbeiter R.Volz; mittlere Schneehöhen und Schneesicherheit (Dezember bis März), von U.Witmer, sowie die Nebelverhältnisse an den Morgenterminen des Winterhalbjahres (September bis März), durch H.Wanner.

Diese kurze Erhebungsspanne von 4 bis 5 Jahren mit einem dichten Beobachtungsnetz (bisweilen über 500 Standorte) wurden mit 10 bis 30 jährigen Reihen des viel lockereren amtlichen Netzes verglichen. Die dieser Arbeit beigelegten Karten basieren auf dem kurzen, dichten Netz. Die phänologischen Karten im Massstab 1:300'000 umfassen 4 Stufen in ansprechender Farbgebung. Auf der Karte der Schneeverhältnisse wurde ein neues, stationbezogenes Symbol in der Form einer zwölfarmigen Schneeflocke (12 Dekaden) gewählt und bewusst auf eine Flächendarstellung verzichtet (1:200'000). Die gleichmassstäbige Nebelkarte unterscheidet in 7 verschiedenen Flächenfarben die Nebelarten und ihre Häufigkeiten.

Diese erfreuliche und fachmännisch ausgewertete und interpretierte grossräumige Klimadarstellung ermöglicht, dem Ziel, den Planern eine klimatologische Wegleitung als weitere Entscheidungshilfe in die Hand zu geben, merklich näher zu kommen.

GH $4 / 80$

Gian A. Gensler, Zürich 